\title{
Using Twitter to investigate opinions about multiple sclerosis treatments: a descriptive, exploratory study [version 1; peer
} review: 2 approved]

\author{
Sreeram V. Ramagopalan ${ }^{\circ}$, Radek Wasiak, Andrew P. Cox
}

Evidera, London, W6 8DL, UK

V1 First published: 10 Sep 2014, 3:216

https://doi.org/10.12688/f1000research.5263.1

Latest published: 10 Sep 2014, 3:216

https://doi.org/10.12688/f1000research.5263.1

\section{Abstract}

Background: Multiple sclerosis (MS) is a common complex disorder, with new treatment options emerging each year. Social media is being increasingly used to investigate opinions about drugs, diseases and procedures. In this descriptive exploratory study, we sought to investigate opinions about currently available MS treatments. Methods: The Twitter resource Topsy was searched for tweets mentioning the following MS treatments: Aubagio, Avonex, Betaferon or Betaseron, Copaxone, Extavia, Gilenya, Lemtrada, Novantrone, Rebif, Tysabri and Tecfidera between 1 Jan 2006 to 31 Jul 2014. Tweets were normalised and sentiment analysis performed.

Results: In total, there were 60037 unique tweets mentioning an MS treatment. About half of the tweets contained non-neutral sentiment. Mean sentiment scores were different for treatments ranging from 0.191 to 0.282 when investigating all tweets. These differences in sentiment scores between treatments were statistically significant $(P<0.001)$. Sentiment scores tended to be higher for oral MS treatments than injectable treatments.

Conclusions: Many tweets about MS treatments have a non-neutral sentiment. The analysis of social media appears to be a potential avenue for exploring patient opinion about MS treatments.

Open Peer Review
Approval Status
version 1
Hobart, Australia
2. Michael Hutchinson, University College
Dublin, Dublin, Ireland
Any reports and responses or comments on the
article can be found at the end of the article.


Corresponding author: Sreeram V. Ramagopalan (sreeram@ramagopalan.net)

Competing interests: SVR, RW and APC are employees of Evidera.

Grant information: The author(s) declared that no grants were involved in supporting this work.

Copyright: @ 2014 Ramagopalan SV et al. This is an open access article distributed under the terms of the Creative Commons Attribution License, which permits unrestricted use, distribution, and reproduction in any medium, provided the original work is properly cited. Data associated with the article are available under the terms of the Creative Commons Zero "No rights reserved" data waiver (CC0 1.0 Public domain dedication).

How to cite this article: Ramagopalan SV, Wasiak R and Cox AP. Using Twitter to investigate opinions about multiple sclerosis treatments: a descriptive, exploratory study [version 1; peer review: 2 approved] F1000Research 2014, 3:216

https://doi.org/10.12688/f1000research.5263.1

First published: 10 Sep 2014, 3:216 https://doi.org/10.12688/f1000research.5263.1 


\section{Introduction}

The analysis of social media is becoming a powerful tool that is being used increasingly to answer research questions across numerous areas including disease spatio-temporal epidemiology and drug adverse events ${ }^{1-3}$. Key stake-holders in the pharmaceutical industry, including patients, physicians, regulatory authorities and pharmaceutical companies, are increasingly using web technologies such as social media, blogs and forums to generate and access opinions and real-world evidence of potentially medically important issues. This content serves as an important source of on-line medical opinions, information and sentiments relating to particular drugs and events. The underlying assumption is that with access to such information, a patient will be able to make more informed decisions about drugs, diseases, procedures and healthcare providers.

Multiple sclerosis (MS) is a chronic, neurodegenerative autoimmune disorder of the central nervous system (CNS). With a prevalence of one per 800 in North America and Northern Europe, MS is the most common acquired neurological disorder in young adults ${ }^{4}$. About $85 \%$ of patients present initially with relapsing-remitting MS (RRMS), characterized by recurrent episodes of neurological dysfunction interspersed with periods of lack of apparent disease activity ${ }^{4}$.

At present, there are nine disease modifying therapies (DMTs) approved by the US Food and Drug Administration (FDA) and 10 DMTs approved by the European Medicines Agency (EMA) for the treatment of RRMS, with new treatment options emerging each year. Approved treatments include interferons (Avonex, Betaferon, Betaseron, Extavia, Rebif), glatiramer acetate (Copaxone), natalizumab (Tysabri), and, more recently, the oral treatments teriflunomide (Aubagio), fingolimod (Gilenya), and dimethyl fumarate (Tecfidera). In this study, we explored whether we could analyse social media to help gauge patient sentiment about treatments using MS as an example. We used the popular social media site Twitter (http://twitter.com) to explore the reporting of patient sentiment and emotions about MS treatments.

\section{Methods}

Data

The Twitter resource, Topsy (http://topsy.com/), which houses all tweets made since 2006, was searched for the following brand names of MS treatments: Aubagio, Avonex, Betaferon or Betaseron, Copaxone, Extavia, Gilenya, Lemtrada, Novantrone, Rebif, Tysabri and Tecfidera using a daily search-time window (i.e. searching for tweets made every day), and specifying the English language. Brand names were used as we thought this would be more likely to reflect patient tweets and further the generic name for some MS treatments are not specific MS treatments. All dates from 1 Jan 2006 to $31 \mathrm{Jul}$ 2014 were searched. For days in which there were more than 1000 tweets satisfying the search criteria, an hourly search-time window was applied for that day, to enable all available tweets to be found (the resource limits searches to 1000 results).

Tweets were downloaded in Extensible Markup Language (XML) format from topsy.com using the application program interface, otterapi (https://code.google.com/p/otterapi/).
Data filtering

Tweets were subsequently filtered to generate datasets for analysis:

1. A unique dataset was generated from the "highlight" data class; thus, removing all directly copied retweets. This was performed so that sentiment analysis could be performed on unique tweets and not bias analyses by having several copies of the same tweet.

All subsequent filtering was case-insensitive.

2. The unique dataset from (1) was filtered to remove items relating to company share prices/stockmarket news.

This was achieved by removing all tweets that contained:

a) "market_jp", "thestreet", "rtebusiness", "pharma", or "pharmsales" in the "permalink" dataclass,

or

b) "bloomberg", "forbes", "dow jones", "financial times", "stockpickr", "marketwatch", "business:", "profit", "shares", or "sec" in the "highlight" data class. This filter was performed as we wanted to identify patient opinion about MS treatments and not stock market related tweets. This filter did retain tweets containing company names, some of which were stock/share price related but some tweets containing company names were from patients.

3. The dataset from (2) was further filtered to remove items that mentioned the manufacturing companies by name: tweets were removed if they contained any of the following:

“novartis", "elan", "biogen", "merck", "bayer", "genzyme", "sanofi", "teva", or "serono". This filter was stringent and removed the majority of stock/share related tweets, but also removed some patient tweets.

\section{Normalisation}

Because of the short nature of tweets, typographical errors, ad-hoc abbreviations, phonetic substitutions, ungrammatical structures and emoticons are common, causing problems for text processing tools. Tokenisation and normalisation to make better sense of the tweet texts was achieved using TwitIE (http://gate.ac.uk/sale/ranlp2013/ twitie/twitie-ranlp2013.pdf?m=1). Normalisation did not remove or alter any of the drug names.

\section{Sentiment and word frequency analysis}

Tweets were grouped into sequential monthly time periods for sentiment analysis using the twitteR R package (https://github.com/ geoffjentry/twitteR/) and Jeffrey Breen's sentiment analysis code (https://github.com/jeffreybreen/twitter-sentiment-analysis-tutorial-201107; a tutorial can be found at: http://www.inside-r.org/howto/ mining-twitter-airline-consumer-sentiment). Word frequency analysis in tweets was performed using TagCrowd (http://tagcrowd.com). TagCrowd uses language-specific lists of common words which are removed from analysis.

\section{Statistical analysis}

Using lists of 2006 positive and 4783 negative words (http://www. cs.uic.edu/ liub/FBS/sentiment-analysis.html\#lexicon), the sentiment score for any tweet is calculated as follows: 
Sentiment score $=$ number of positive words - number of negative words

If the sentiment score $>0$, this means that the sentence has an overall 'positive opinion', if the sentiment score $<0$, this means that the sentence has an overall 'negative opinion', if the sentiment score $=0$, then the sentence is considered to be a 'neutral opinion'. Sentiment scores were summed for all tweets for each MS treatment, and

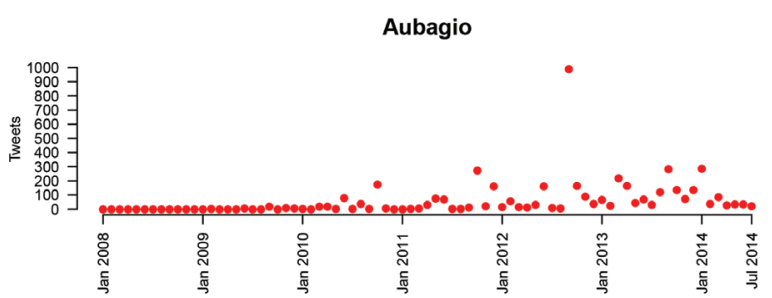

Betaferon

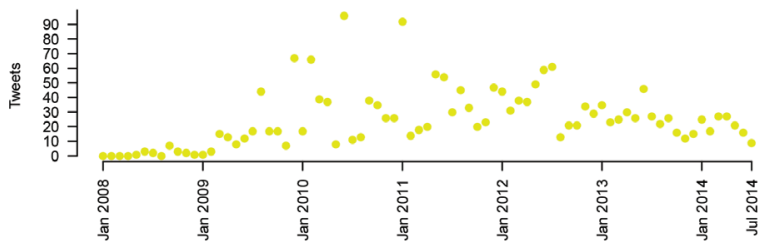

Extavia

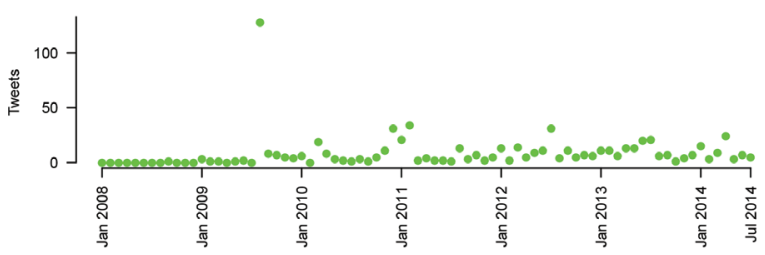

Lemtrada

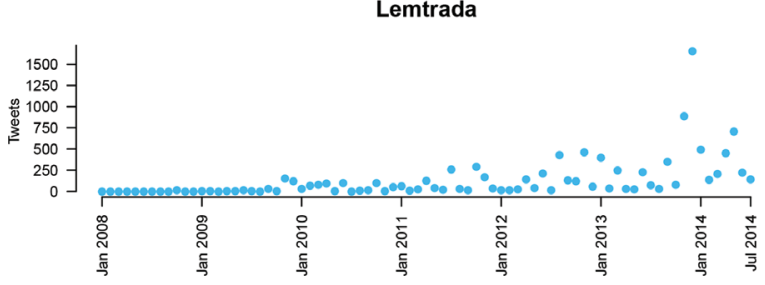

Rebif
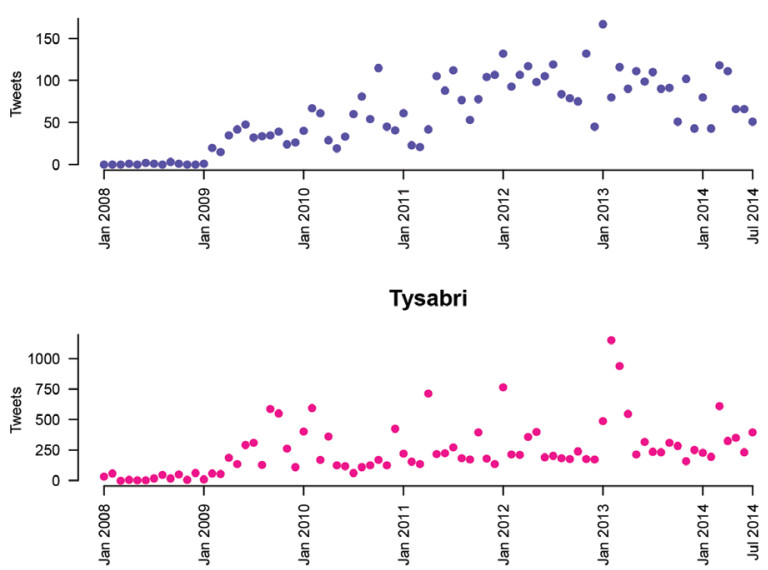

Figure 1. Number of tweets by month for each treatment. means calculated. Mean sentiment scores were compared across treatments using the Kruskal-Wallis test. Statistical analysis was performed using $\mathrm{R}$ version 3.1 .1 and $\mathrm{p}$ values less 0.05 were considered significant.

\section{Results}

In total, there were 60037 unique tweets mentioning an MS treatment. The number of tweets by month is shown in Figure 1. Tweets
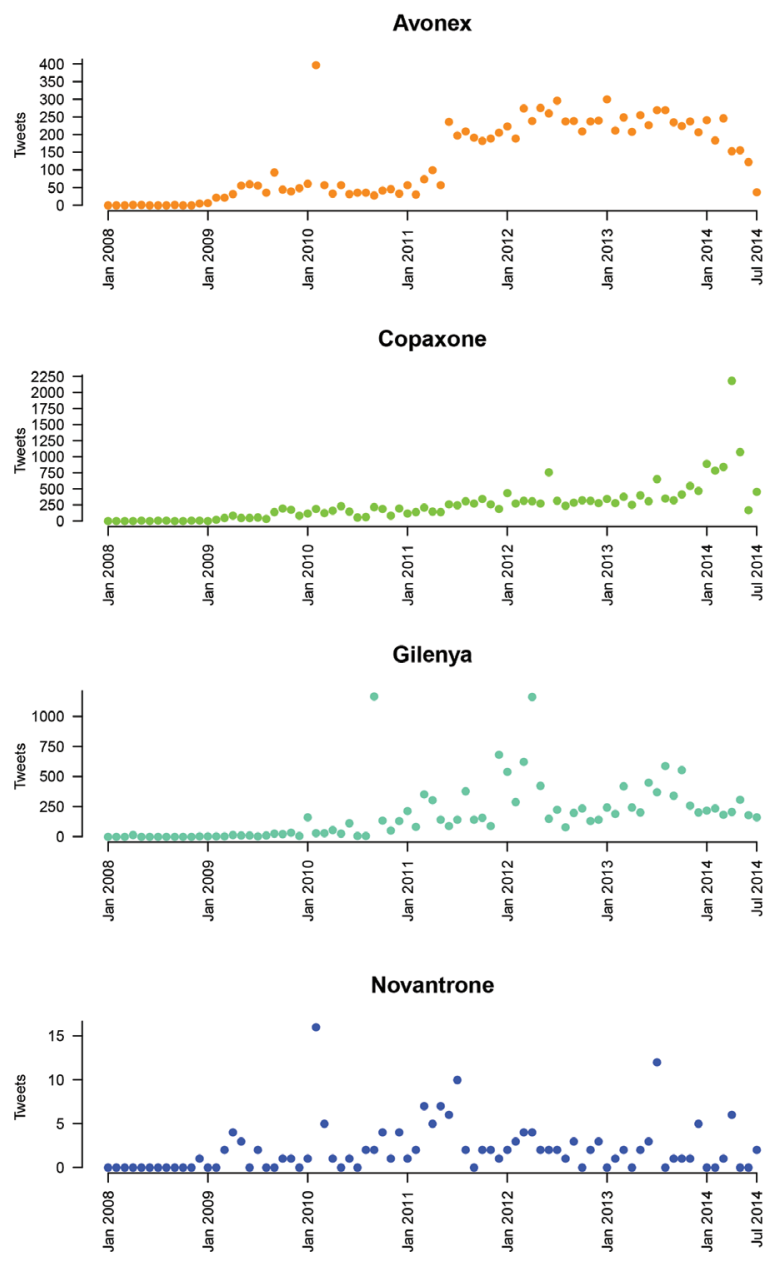

Tecfidera

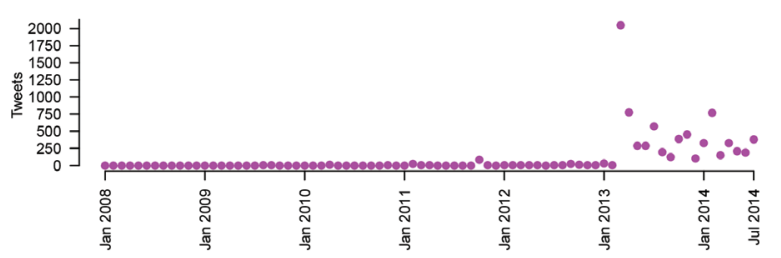


for Tysabri started the earliest (January 2008) and Aubagio the latest (February 2009). When removing tweets that included share/stock information there were 56708 unique tweets and when removing tweets that included share/stock information or company names there were 41690 unique tweets.

The number of tweets by treatment, overall and when removing tweets that included share/stock information and/or company names is shown in Table 1. Tysabri had the largest number of tweets ( $n=14542$, all tweets; $n=10984$ after filtering for company names and stock/share tweets) and Novantrone had the lowest ( $\mathrm{n}=110$, all tweets; $n=109$ after filtering for company names and stock/share tweets), both before and after filtering.

The sentiment score analysis of all normalised tweets, normalised tweets excluding those that contained share/stock information and normalised tweets excluding those that contained share/stock information and company names are shown in Table 2, Table 3 and Table 4. About half of all tweets in all analyses had a neutral sentiment (43-61\%, all tweet data; 45-57\% after filtering for company names and stock/share tweet data). Tweets for drugs that contained sentiment were more likely to be positive sentiment, apart from tweets for Novantrone and Tysabri (23-33\% for drugs apart from Novantrone $(16 \%$ ), all tweet data; $24-31 \%$ for drugs apart from Novantrone (17\%) and Tysabri (28\%), after filtering for company names and stock/share tweet data).

Summing sentiment scores for all tweets showed positive overall sentiment scores for all drugs apart from Novantrone (all analyses) and Tysabri (only after filtering for company names and stock/share tweet data). Gilenya had the highest summed sentiment score in all analyses. Boxplots of sentiment scores of all normalised tweets, normalised tweets excluding those that contained share/stock information and normalised tweets excluding those that contained share/ stock information and company names are shown in Figure 2, Figure 3 and Figure 4. The mean sentiment score ranged from -0.191 to 0.282 (all tweet data); and -0.193 to 0.247 (after filtering for company names and stock/share tweet data). Novantrone always had the lowest mean sentiment score. Tecfidera had the highest mean score in the all tweet data, and Aubagio had the highest mean score in the filtered for company names and stock/share tweet data. The mean sentiment scores were different in all analyses $(\mathrm{P}<0.001$ in the all tweet data, filtered for stock/share tweet data and filtered for company names and stock/share tweet data).

Most common words in tweets for treatments were investigated. Example word clouds for the 50 most common words (excluding commonly used English words and drug names) in all normalised tweets for Avonex, Rebif and Tysabri are shown in Figure 5, Figure 6 and Figure 7. Of note is the frequency of 'flu' and 'injection' in Avonex and Rebif tweets and 'infusion' and 'pml' in Tysabri tweets.

\section{Discussion}

We present here, to the best of our knowledge, the first analysis of social media for MS treatments. A significant proportion of tweets did contain non-neutral sentiment about MS treatments, and the distribution of sentiment score was different between treatments. Thus it appears that Twitter can be a potential resource to understand patient opinion about MS treatments. When looking at frequency of words, notably 'flu' and 'injection' were in the 50 most common words in tweets about Rebif and Avonex and 'infusion' and 'pml' in the 50 most common words in tweets about Tysabri. Flu-like

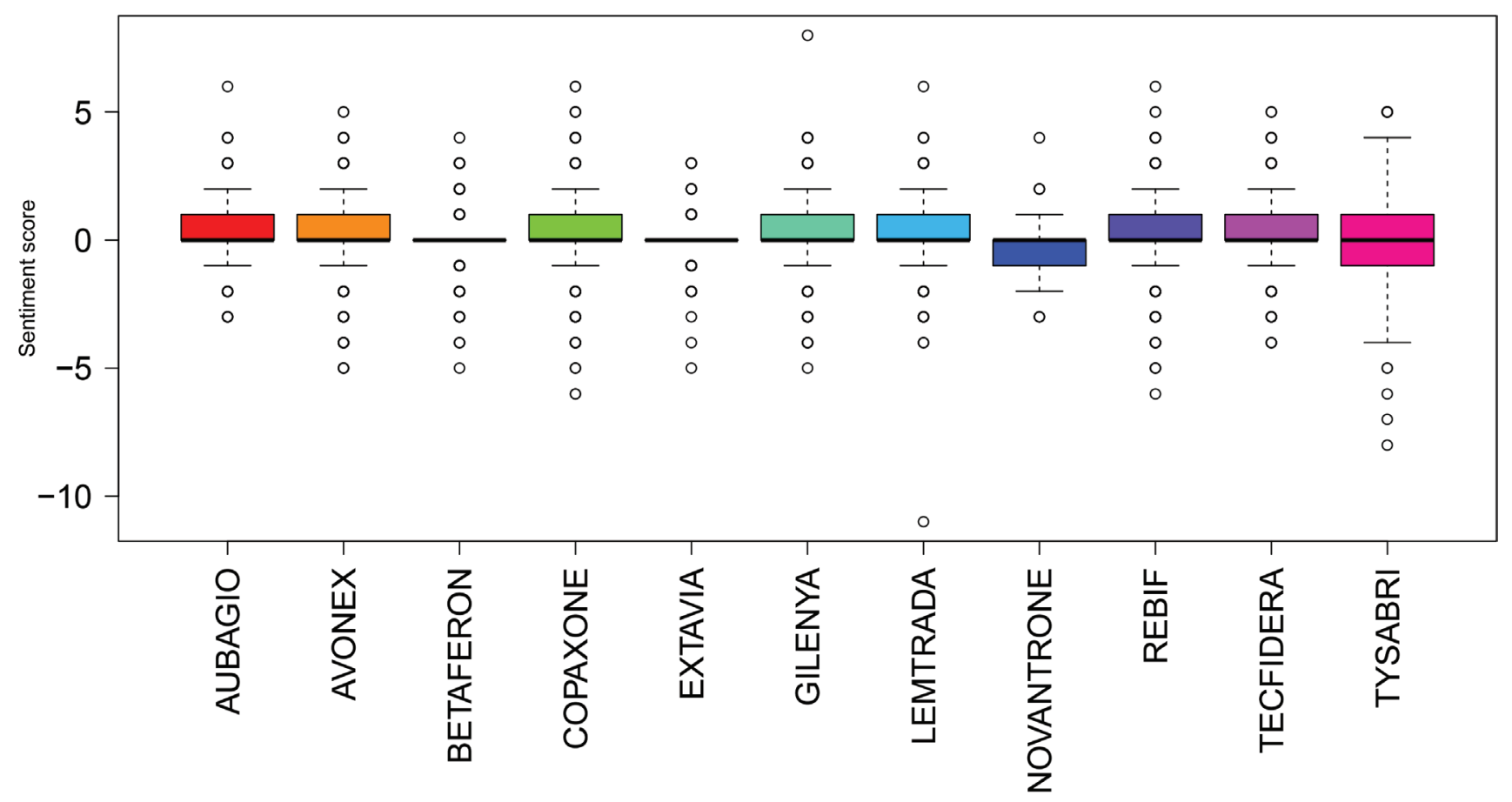

Figure 2. Boxplots of sentiment scores for all normalised tweets. 


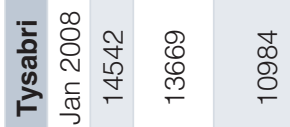

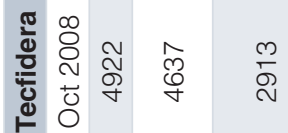

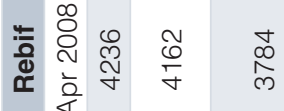

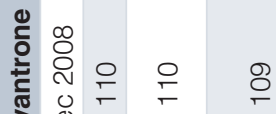

วั้

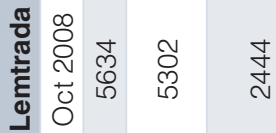

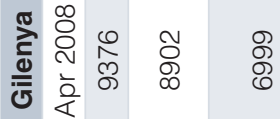

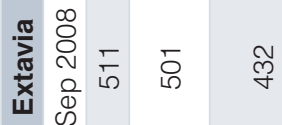

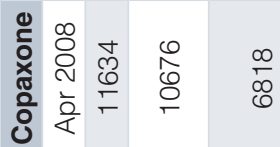

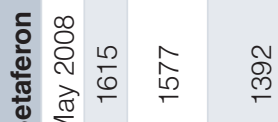

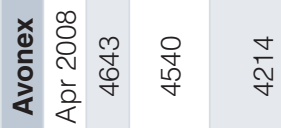

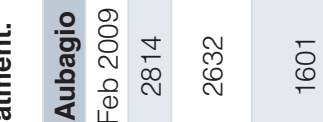

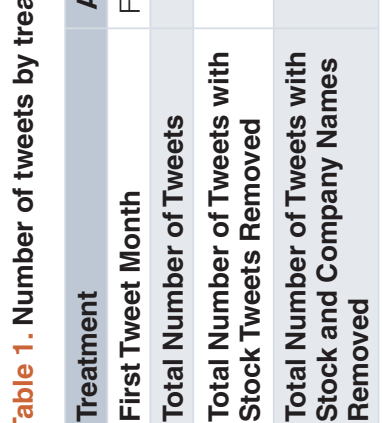

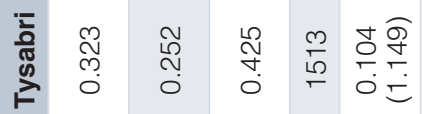

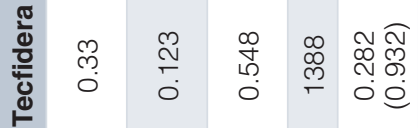

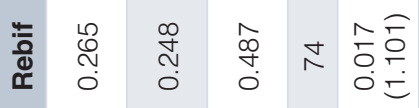

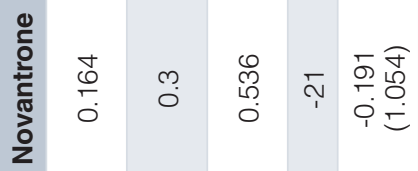

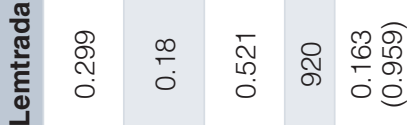

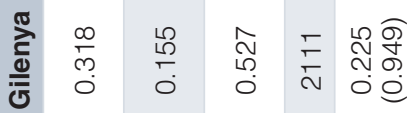

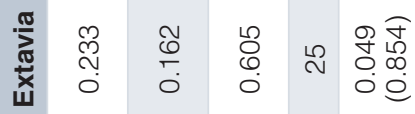

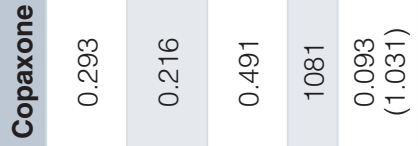

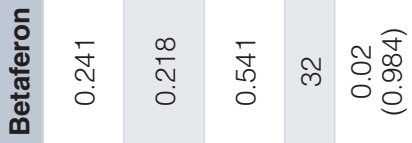

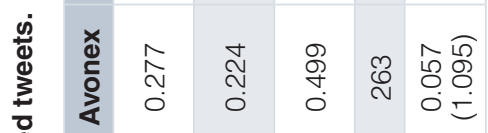

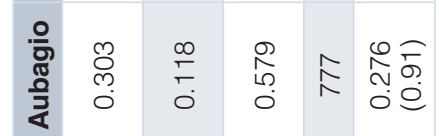

高

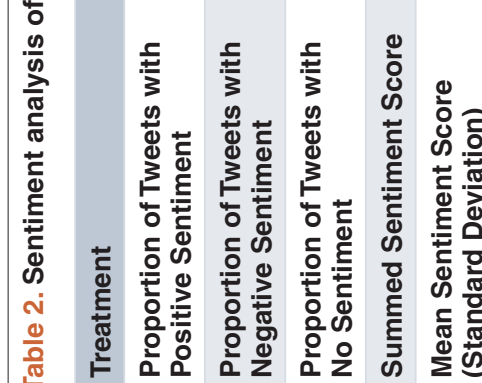

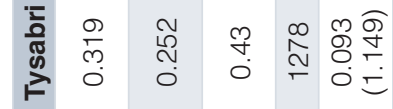

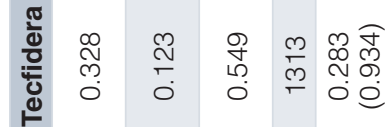

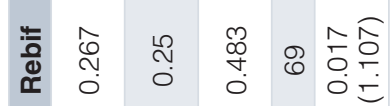

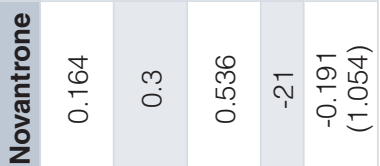

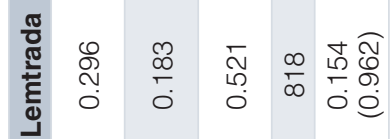

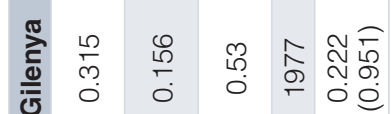

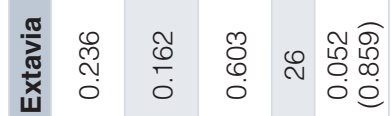

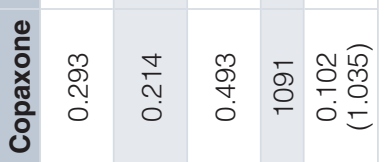

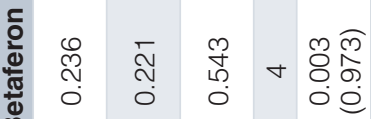

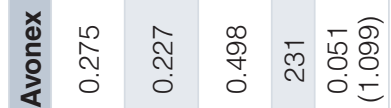

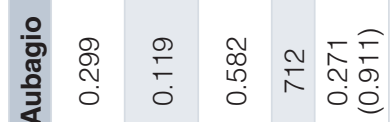

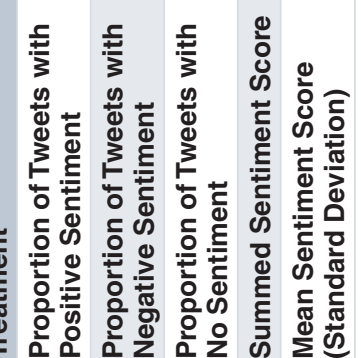

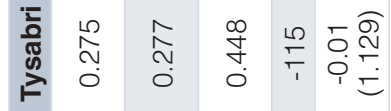

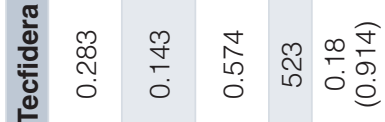

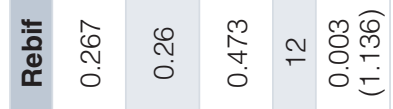

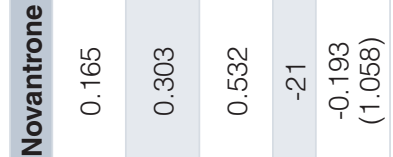

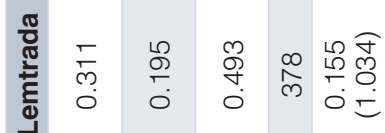

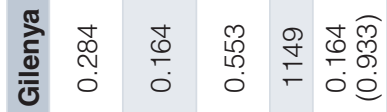

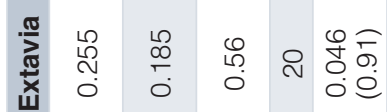

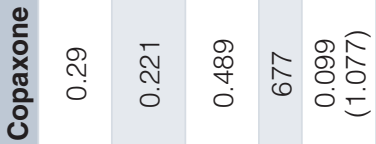

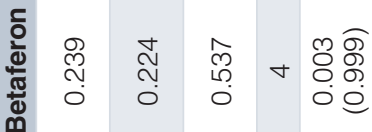

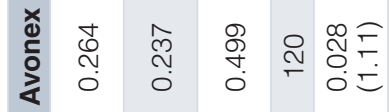

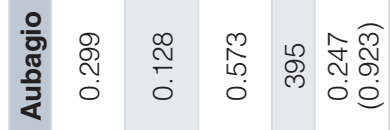

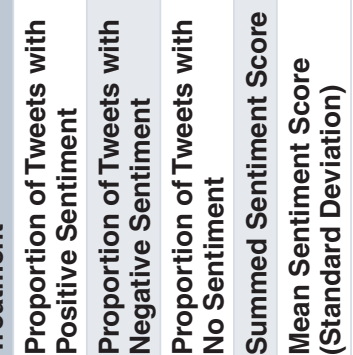




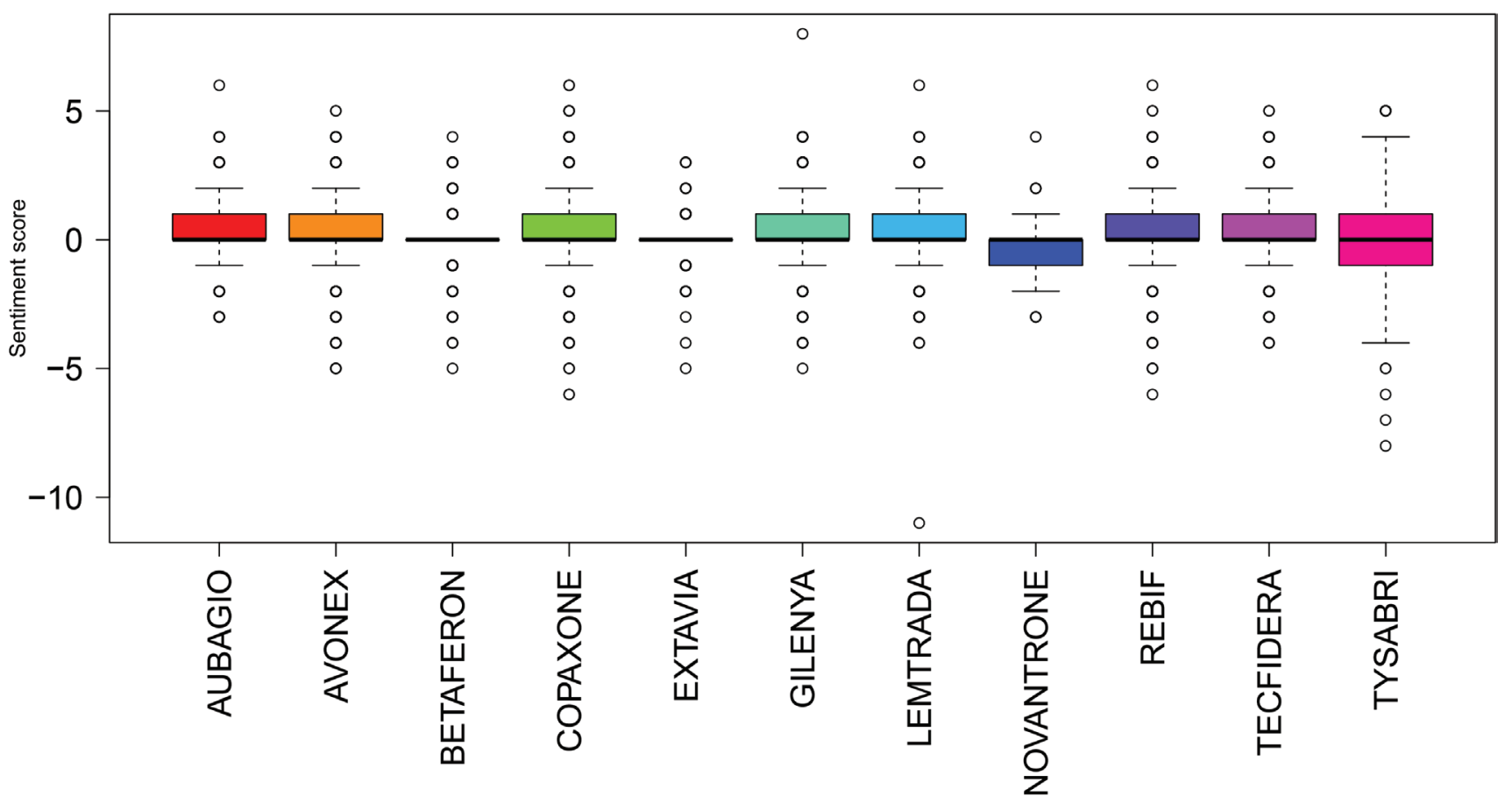

Figure 3. Boxplots of sentiment scores of all normalised tweets with tweets containing share/stock information excluded.

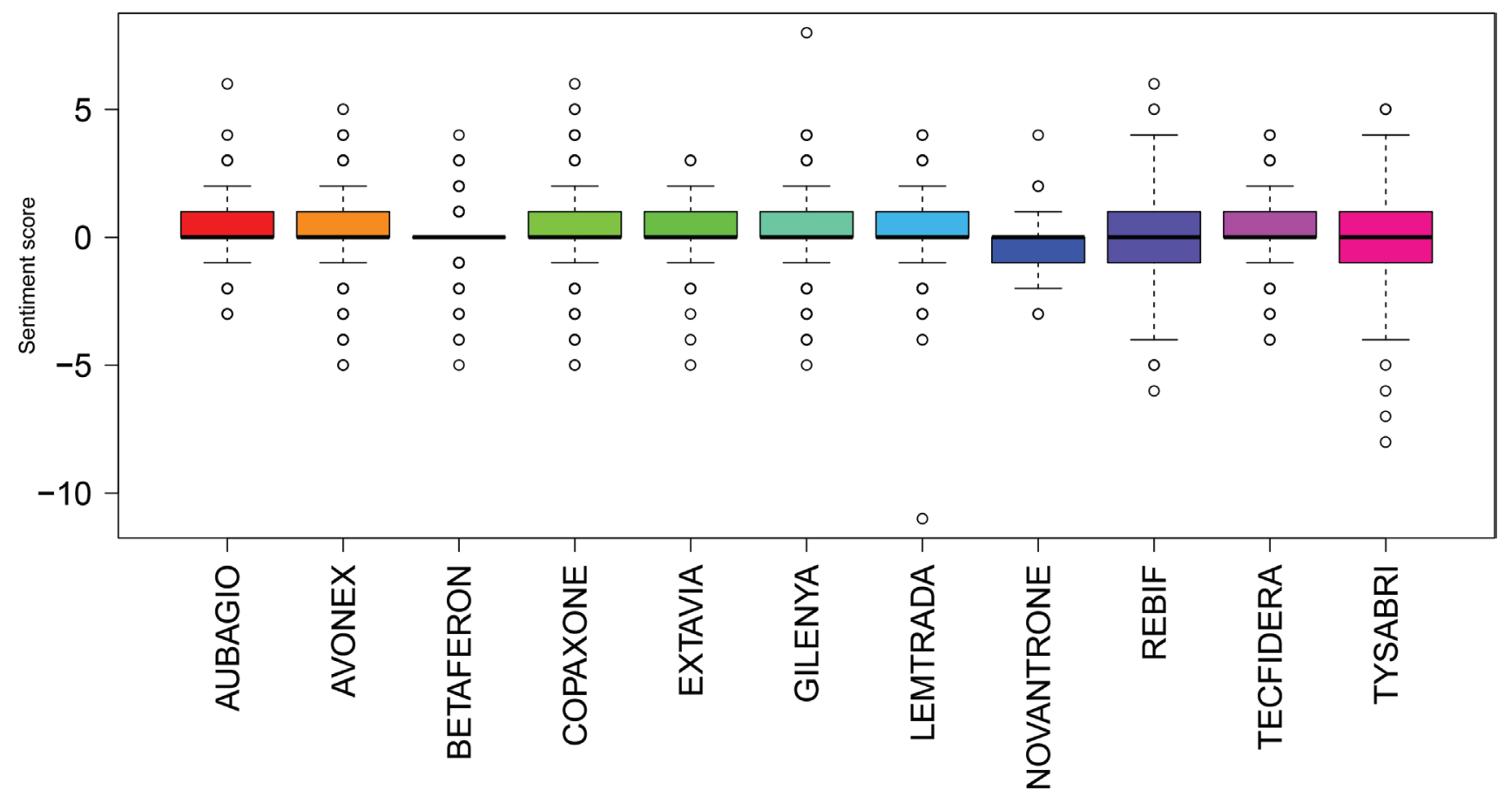

Figure 4. Boxplots of sentiment scores of all normalised tweets with tweets containing share/stock information and company names excluded. 


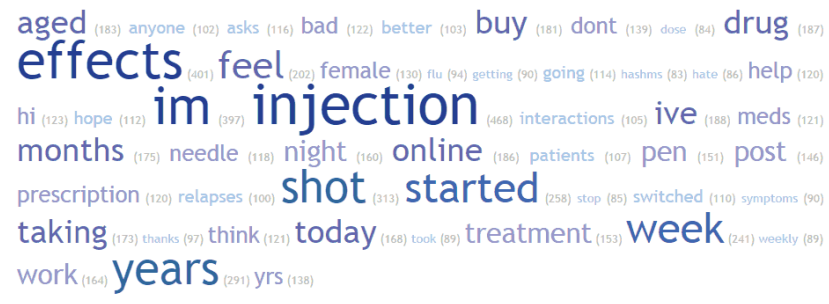

Figure 5. Word cloud for all normalised tweets for Avonex.

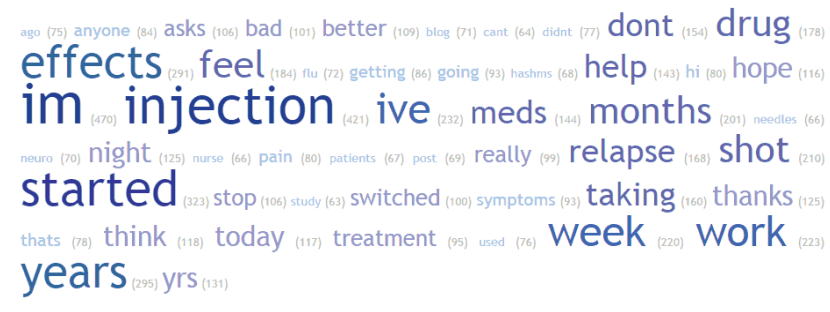

Figure 6. Word cloud for all normalised tweets for Rebif.

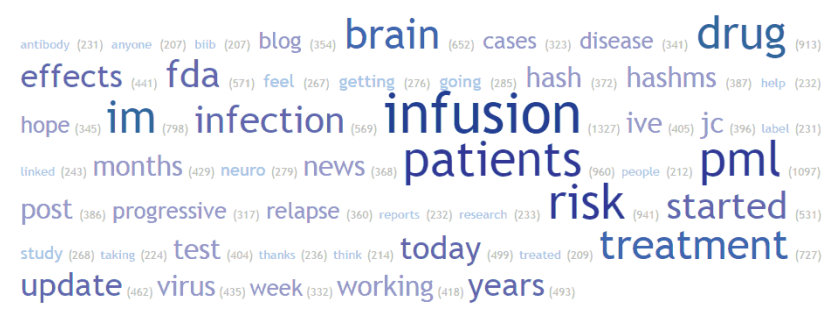

Figure 7. Word cloud for all normalised tweets for Tysabri.

symptoms are well known side-effects of the injectible treatments Rebif and Avonex and progressive multifocal leukoencephalopathy or 'pml', is a well-known risk for patients taking the intravenously infused Tysabri ${ }^{5}$. This provides some sort of face validity for our results reflecting real specific tweets about MS treatments.

Interestingly, the oral MS treatments- Gilenya, Aubagio and Tecfidera had the highest mean sentiment scores and Gilenya had the highest summed sentiment score in all analysis. This may reflect a well known patient preference for oral therapies as compared to injectible treatments ${ }^{6,7}$. Further work is needed to explore tweets in detail to see if the higher mean sentiment scores are related to positive tweets about the fact that these drugs are to be taken orally.

There are a number of limitations to this study. We are using automated tools to assign sentiment to tweet content- these tools will not recognise the intricacies of human language e.g. the context of the tweet and sarcasm for example. Further, whilst we tried to normalise tweets, the diversity of twitter slang will mean that abbreviations may not be recognised. We may have underestimated the number of tweets as we used brand names to identify drugs. Any tweets using the generic name or shortened versions will be missed. Whilst we tried to focus on tweets from patients, it is inevitable that business related tweets will have been included in our analysis and some patient tweets lost during filtering. It is also possible that not all tweets were delivered to us by the Twitter interface, although that is not possible to verify.

Our findings and any interpretation should be regarded as speculative and exploratory. The results represent what can be potentially done relatively quickly and easily using data from Twitter. More rigorous analytical methods can be applied for more specific questions (e.g. the analysis of adverse events). It is clear from this study that tweets are written about MS treatments and many of these have a non-neutral sentiment. Further work is needed to look at these tweets in detail to further understand patient opinion about MS treatments.

\section{Author contributions}

SVR is the guarantor of the study. SVR performed the analysis. APC contributed to the analysis and interpretation of the data. SVR wrote the first draft and all authors contributed to subsequent drafts and the final paper.

\section{Competing interests}

SVR, RW and APC are employees of Evidera.

Grant information

The author(s) declared that no grants were involved in supporting this work.
1. Broniatowski DA, Paul MJ, Dredze M: National and local influenza surveillance through Twitter: an analysis of the 2012-2013 influenza epidemic. PLoS One. 2013; 8(12): e83672.

PubMed Abstract | Publisher Full Text | Free Full Text

2. Tsuya A, Sugawara $Y$, Tanaka A, et al:: Do cancer patients tweet? Examining the twitter use of cancer patients in Japan. J Med Internet Res. 2014; 16(5): e137. PubMed Abstract | Publisher Full Text | Free Full Text

3. Freifeld CC, Brownstein JS, Menone CM, et al.: Digital drug safety surveillance: monitoring pharmaceutical products in twitter. Drug Saf. 2014; 37(5): 343-350.

PubMed Abstract | Publisher Full Text | Free Full Text

4. Ramagopalan SV, Dobson R, Meier UC, et al:: Multiple sclerosis: risk factors, prodromes, and potential causal pathways. Lancet Neurol. 2010;
9(7): 727-739

PubMed Abstract | Publisher Full Text

5. Tanasescu R, lonete $\mathrm{C}$, Chou IJ, et al.: Advances in the treatment of relapsingremitting multiple sclerosis. Biomed J. 2014; 37(2): 41-49.

PubMed Abstract | Publisher Full Text

6. Dibonaventura MD, Wagner JS, Girman GJ, et al.: Multinational Internet-based survey of patient preference for newer oral or injectable Type 2 diabetes medication. Patient Prefer Adherence. 2010; 4: 397-406.

PubMed Abstract | Publisher Full Text | Free Full Text

7. Fallowfield L, Atkins L, Catt S, et al.: Patients' preference for administration of endocrine treatments by injection or tablets: results from a study of women with breast cancer. Ann Oncol. 2006; 17(2): 205-210.

PubMed Abstract | Publisher Full Text 


\section{Open Peer Review}

\section{Current Peer Review Status:}

\section{Version 1}

Reviewer Report 09 December 2014

https://doi.org/10.5256/f1000research.5610.r6809

(C) 2014 Hutchinson M. This is an open access peer review report distributed under the terms of the Creative Commons Attribution License, which permits unrestricted use, distribution, and reproduction in any medium, provided the original work is properly cited.

\section{Michael Hutchinson}

St Vincent's University Hospital, University College Dublin, Dublin, Ireland

An interesting paper and an introduction to the reviewer of the new methods of analysis of social media. The authors have emphasised the pains that they took to exclude tweets from company and financial sources. The difficulty is that one cannot be certain that what they are analysing is not contaminated by tweets stimulated in some way from particular pharmaceutical companies. There is, of course, a "newness" affect and this explains the frequency of the tweets for Aubagio and Tecfidera. If one excludes Novantrone and Tysabri, then the lowest sentiment scores are for the interferons. What I find remarkable in this report is that the highest mean sentiment score is for Aubagio, a drug which many neurologists would have less enthusiasm for, given problems in its use in patients of childbearing age. Interesting findings and nicely presented paper, but I am still concerned about the source data; are all these tweets from genuine patients?

Competing Interests: Disclosures: Michael Hutchinson served on a medical advisory board for the CONFIRM study [BG00012] for Biogen Idec, serves on the editorial board of the Multiple Sclerosis Journal, has received speaker's honoraria from Merck- Serono, Novartis, Biogen Idec and BayerSchering and receives research support from Dystonia Ireland, the Health Research Board of Ireland and the Foundation for Dystonia Research.

\section{I confirm that I have read this submission and believe that I have an appropriate level of} expertise to confirm that it is of an acceptable scientific standard.

Reviewer Report 28 November 2014

\section{https://doi.org/10.5256/f1000research.5610.r6808}

(C) 2014 Taylor B. This is an open access peer review report distributed under the terms of the Creative Commons Attribution License, which permits unrestricted use, distribution, and reproduction in any medium, provided the original work is properly cited. 


\section{Bruce V Taylor}

Menzies Research Institute Tasmania, University of Tasmania, Hobart, Australia

I found this a very interesting report and feel that as the authors do that this method of analysis may provide a real world window into patient perceptions of treatment, efficacy, and side effects. It would be interesting to assess churn in the twitter responses, for example PML in Tysabri is a major issue to patients and it would be interesting to see how this has trended over time, as the period for which tweets were collected includes the period of time when PML was a major issue (newly described) and would have been a major source of negative sentiment. Similarly for the newer agents it would be interesting to track these findings over time from release date and see whether positivity or negativity changes with patient experience.

This type of research will undoubtedly provide significant and important patient focused outcomes for future medication usage and tolerance studies. at considerable cost efficiency.

Can the authors look at trending words in these tweets, for example PML in Tecfidera or hair loss for Aubagio?

Competing Interests: No competing interests were disclosed.

\section{I confirm that I have read this submission and believe that I have an appropriate level of expertise to confirm that it is of an acceptable scientific standard.}

The benefits of publishing with F1000Research:

- Your article is published within days, with no editorial bias

- You can publish traditional articles, null/negative results, case reports, data notes and more

- The peer review process is transparent and collaborative

- Your article is indexed in PubMed after passing peer review

- Dedicated customer support at every stage

For pre-submission enquiries, contact research@f1000.com

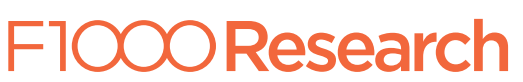

